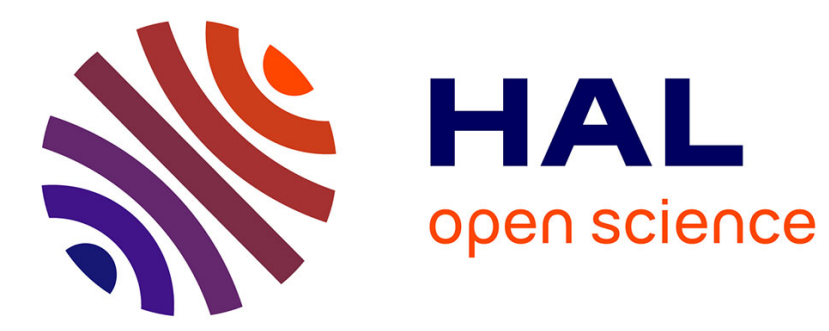

\title{
Femtosecond time-resolved Electronic Relaxation Dynamics in Tetrathiafulvalene
}

David Staedter, Nicolas Thiré, Laurent Polizzi, Y Mairesse, P Mayer, Valérie Blanchet

\section{- To cite this version:}

David Staedter, Nicolas Thiré, Laurent Polizzi, Y Mairesse, P Mayer, et al.. Femtosecond timeresolved Electronic Relaxation Dynamics in Tetrathiafulvalene. Journal of Chemical Physics, 2015, 142 (19), pp.194306. 10.1063/1.4921319 . hal-01082495

\section{HAL Id: hal-01082495 \\ https://hal.science/hal-01082495}

Submitted on 24 Nov 2014

HAL is a multi-disciplinary open access archive for the deposit and dissemination of scientific research documents, whether they are published or not. The documents may come from teaching and research institutions in France or abroad, or from public or private research centers.
L'archive ouverte pluridisciplinaire HAL, est destinée au dépôt et à la diffusion de documents scientifiques de niveau recherche, publiés ou non, émanant des établissements d'enseignement et de recherche français ou étrangers, des laboratoires publics ou privés. 


\title{
Femtosecond time-resolved Electronic Relaxation Dynamics in Tetrathiafulvalene
}

\author{
D. Staedter, ${ }^{1}$ N. Thiré, ${ }^{1,2}$ L. Polizzi, ${ }^{1}$ Y. Mairesse, ${ }^{3}$ P. Mayer, ${ }^{4}$ and V. Blanchet ${ }^{1,3, a)}$ \\ ${ }^{1)}$ Laboratoire de Collisions, Agrégats et Réactivité, IRSAMC, Univ. de Toulouse-CNRS, \\ 31062 Toulouse, France \\ 2) INRS-EMT, Advanced Laser Light Source, 1650 Lionel-Boulet Bvd, \\ Varennes, J3X1S2 Canada \\ ${ }^{3)}$ Centre des Laser Intenses et Applications, Univ. de Bordeaux-CNRS, \\ 33405 Talence, France \\ ${ }^{4)}$ Chemistry Department, University of Ottawa, \\ Ottawa, Canada K1N $6 N 5$
}

(Dated: 13 November 2014)

In the present paper the ultrafast electronic relaxation of tetrathiafulvalene (TTF) initiated around $4 \mathrm{eV}$ is studied for the first time by femtosecond time-resolved velocity-map imaging. The goal is to enlighten the broad double structure observed in the absorption spectrum at such energy. By monitoring the transients of the parent cation and its fragments and by varying both the pump and probe wavelengths, two internal conversions are detected with typical time constants of $260 \mathrm{fs}$ and of $600 \mathrm{fs}$ as well as intramolecular vibrational relaxation. Photoelectron images strengthen the analysis of the relaxation process. In addition the formation of the dimer of TTF has been revealed.

PACS numbers: $33.15 \mathrm{Vb}, 33.50$. Hv, $33.60+\mathrm{q}, 33.80-\mathrm{b}$

Keywords: Electronic relaxation, multiphoton ionization

\section{INTRODUCTION}

Since the discovery of the unusual electrical conductance in organic materials based on tetrathiafulvalene $\left(\mathrm{TTF}, \mathrm{C}_{6} \mathrm{~S}_{4} \mathrm{H}_{4}\right)$ in $1972,{ }^{1}$ and metal TCNQ-TTF in $1973,{ }^{2}$, TTF has been among the most studied heterocyclic systems. TTF and its derivatives are well known as efficient $\pi$ electron-donors ${ }^{3}$ with many uses in material chemistry. $^{4-8}$ The conductivity of the non-aromatic 14 $\pi$ electron system with four discoordinated sulfur atoms has been rationalized by its small isomerization barrier $\left(\sim 40 \mathrm{~cm}^{-1}\right)$ from the nonplanar boat-like equilibrium structure of the neutral $\left(C_{2 v}\right.$ symmetry) to a planar conformation in its cation $\left(D_{2 h}\right.$ symmetry). ${ }^{9-11}$ Both, the higher degree of aromaticity reached by the coupling between the electron removal and the 'boat' deformation and the metallic behavior enhancement with the intermolecular S-S contacts have been suggested to play a role in the mechanism of superconductivity. ${ }^{10}$

One of the fundamental issues in designing molecular systems for photoinduced charge separation is to investigate the primary photophysical relaxation. Spectral studies of fullerenes functionalized by TTF derivatives have been reported in the ultraviolet range (UV), ${ }^{12}$ as well as picosecond-resolved absorption spectra. ${ }^{13,14}$ Both, the donor-acceptor system and the oligothiophene alone in liquid phase has been studied in femtosecond time-resolved spectroscopy. ${ }^{15}$ For many of these photoactive compounds, formed by Van der Waals polarization interactions, ${ }^{12}$ the Highest Occupied Molecular Orbital

\footnotetext{
a)Electronic mail: blanchet@celia.u-bordeaux1.fr
}

(HOMO) is mostly localized on the TTF moiety and the lowest unoccupied molecular orbital on the acceptor moiety. ${ }^{16}$ The photoexcitation in the visible range triggers a fast energy transfer from TTF onto the acceptor moiety before the appearance of a charge transfer on the picosecond time-scale. ${ }^{16}$ In the UV range it could be slightly different due to an efficient electronic relaxation within the TTF moiety alone.

The TTF absorption spectrum, shown in Figure 1, exhibits two close-lying intense peaks at around 317 and $303 \mathrm{~nm}^{17-20}$ which are still without a clear assignment. It is within this framework, that the present pump-probe study of the TTF moiety alone is investigating dynamics initiated around $4 \mathrm{eV}(\approx 310 \mathrm{~nm})$ by time-resolved photoionization in the gas phase. The lowest energy transitions both in TTF and $\mathrm{TTF}^{+}$have been calculated by different techniques ${ }^{21}$ and among others by time-dependent density functional theory (TD-DFT). ${ }^{22-24}$ The photoelectron spectrum of TTF has been recorded by several groups reporting a vertical ionization energy, $\mathrm{IP}_{1}=6.7 \pm$ $0.02 \mathrm{eV}$ and a first excited cation state at $\mathrm{IP}_{2}=8.58 \pm 0.02$ $\mathrm{eV}^{25-28}$ The dissociative ion continuum that starts $\sim 10$ $\mathrm{eV}$ has been studied recently up to $13 \mathrm{eV} .^{29}$

Following the IUPAC convention, the $(x, y)$ axis defines the $\mathrm{C}_{2} \mathrm{~S}_{4}$ plane with the y-axis parallel to the central $\mathrm{C}=\mathrm{C}$ double bond (long axis) in the $C_{2 v}$ geometry. On the contrary, in the $D_{2 h}$ geometry, the molecular plane is now $(y, z)$ with the $\mathrm{z}$-axis parallel to the long axis. By TD-DFT calculations converging to a $C_{2 v}$ boat geometry, Fabian et al. assigned the weak absorption band around $368 \mathrm{~nm}$ to a $A_{1} \rightarrow B_{1}$ transition $\left(\pi \rightarrow \pi^{*}\right.$ along $\mathrm{x})$ and found only one state lying in the intense double structure $(303 / 317 \mathrm{~nm})$ corresponding to a $A_{1} \rightarrow B_{2}$ transition $\left(\pi \rightarrow \pi^{*}\right.$ along y). ${ }^{30}$ The oscillator strengths 
(a)

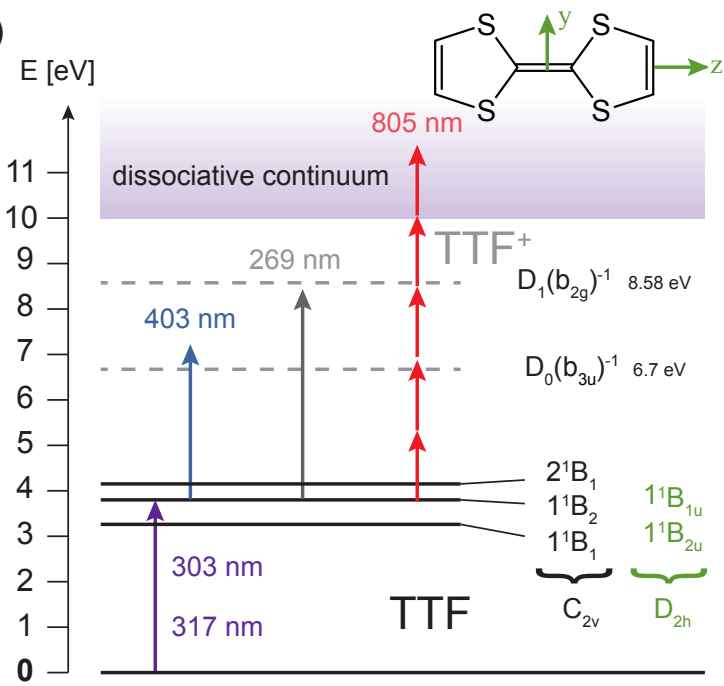

(b)

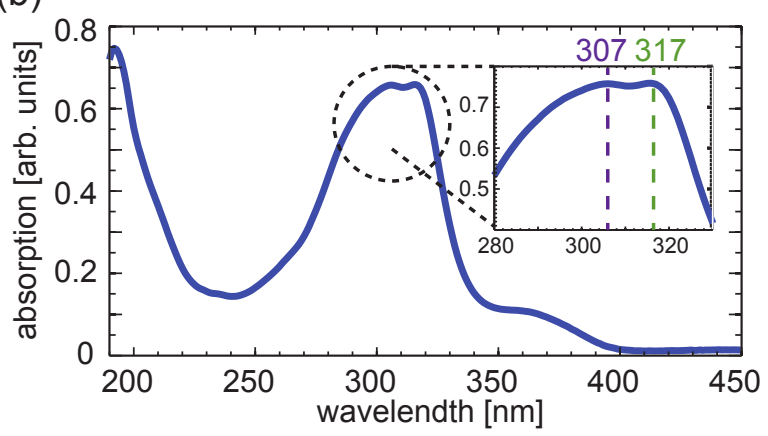

FIG. 1. (a) The different pump-probe excitation schemes used. The definition of $y$ and $z$ axis is relevant only for the $\mathrm{D}_{2 h}$ planar geometry. (b) Absorption spectrum of TTF in acetonitrile at ambient temperature with a concentration of $5 \times 10^{-4} \mathrm{~mol} / \mathrm{l}$.

calculated by this TD-DFT study at $368 \mathrm{~nm}$ and $\approx 307$ $\mathrm{nm}$ are very similar, which goes strongly against the experimental observations in Figure 1.

Andreu et al., as well by TD-DFT calculations, still in the $\mathrm{C}_{2 v}$ geometry, assigned the two most intense excitations to the $1^{1} B_{2}$ state transition, described mainly by the $\mathrm{HOMO}$ to $\mathrm{LUMO}+5\left(\mathrm{~b}_{2}\right.$, antibonding orbital $)$ transition at $317 \mathrm{~nm}$ and a second one at $303 \mathrm{~nm}$ upto the $2^{1} B_{1}$ state described mainly by the HOMO to LUMO $+4\left(b_{1}\right.$, bonding orbital) transition. ${ }^{23}$ These calculations match the experimental oscillator strengths as well as the polarizations much better than the ones from Fabian et al. It is this notation that has been used in Figure 1. In this work, the weak absorption band around $368 \mathrm{~nm}$, assigned as well to a $1^{1} B_{1}$ state, corresponds to the first $\pi \rightarrow \pi^{*}$ transition with a $\operatorname{HOMO}\left(\mathrm{a}_{1}\right)$ to the $\mathrm{LUMO}+1\left(\mathrm{~b}_{1}\right)$ excitation, while the HOMO to the LUMO $+2\left(\mathrm{a}_{2}\right)$ excitation is symmetry forbidden. ${ }^{23}$

The TD-DFT calculation done by Pou-Armengo, still in $\mathrm{C}_{2 v}$ geometry, differs from Andreu et al; in addition to the $1^{1} B_{2}$ and $2^{1} B_{1}$ states, they found a $2^{1} B_{2}$ state at $4.27 \mathrm{eV}(290 \mathrm{~nm})$ with an oscillator strength seven times larger than the two lower states. ${ }^{22}$ Finally, these same authors did as well a multistate extension of a multiconfigurational second-order perturbation method (MSCASPT2) calculation ${ }^{21}$ with an active electron space that includes the $3 \mathrm{~s}, 3 \mathrm{p}$, and $3 \mathrm{~d}$ orbitals and a TD-DFT calculation, ${ }^{22}$ both in a $D_{2 h}$ optimized geometry. Using this symmetry is validated by the weak isomerization barrier towards the $C_{2 v}$ symmetry. In the most precise MS-CASPT2 calculation, they predict around $406 \mathrm{~nm}$, a weak transition $1^{1} A_{g} \rightarrow 1^{1} B_{2 u}$ polarized along the shortaxis ( $x$ in the $C_{2 v} \rightarrow y$ in $D_{2 h}$ ) and around $309 \mathrm{~nm}$, an intense transition polarized along the long axis ( $y$ in the $C_{2 v} \rightarrow z$ in the $\left.D_{2 h}\right)$ to the $1^{1} B_{1 u}$ state. From both calculations, the authors consequently conclude that the double structure at $\sim 310 \mathrm{~nm}$ reveals a vibrational structure in the $1^{1} B_{1 u}$ band ${ }^{21,31}$ and not an electronic structure. The central $\mathrm{C}-\mathrm{C}$ vibrational mode is indeed around 1600 $\mathrm{cm}^{-132}$ and in good agreement with the energy splitting of $1460 \mathrm{~cm}^{-1}$ observed on the absorption spectrum between the two maxima. The $1^{1} B_{1 u}$ electronic excitation is defined at $86 \%$ by an singly excited configuration of a $\mathrm{HOMO}$ to $\mathrm{LUMO}+4\left(4 \mathrm{~b}_{2 g}\right)$ transition. In this calculation, the Rydberg states have been predicted as low as $3.63 \mathrm{eV}$.

In the present experiment, by scanning the central pump wavelength between 307 and $322 \mathrm{~nm}$, we aim to investigate if the induced dynamics change drastically through this intense UV band. To enhance the sensitivity, the photoionization is achieved at different probe wavelengths $(267 \mathrm{~nm}, 398 \mathrm{~nm}$ and $800 \mathrm{~nm})$. In addition the photoelectron spectra are recorded. In what follows, the multiphoton pump-probe pathway is denoted as (a $\left.+b^{\prime}\right)$, where a and b' indicate the number of absorbed pump and probe photons, respectively, and the prime designates the probe. The remainder of the paper starts with a description of the experimental setup in section 2 . Next, the time-resolved signals are presented as a function of each probe wavelengths in section 3 . Finally, the results are discussed in section 4 .

\section{EXPERIMENTAL SET-UP}

The pump and probe pulses are generated from a 1 $\mathrm{kHz}-1.5 \mathrm{~mJ}$ regenerative amplifier centered at $795 \mathrm{~nm}$ delivering a fundamental pulse with a Fourier limited Full-Width at Half Maximum (FWHM) of 70 fs. A 400 nm-pumped Non-collinear Optical Parametric Amplifier (NOPA) with three-steps of amplification and a double pass prisms compressor provide a $30 \mu \mathrm{J} / \mathrm{p}$ pulse, tunable between 500 and $700 \mathrm{~nm}$. The duration is measured by autocorrelation via a $50 \mu \mathrm{m}$ BBO-I crystal to $\approx 80 \mathrm{fs}$. By a subsequent second harmonic generation ( $\mathrm{SHG}$ ) of the output of the NOPA or a sum frequency mixing (SFG) with the fundamental through a $200 \mu \mathrm{m}$ BBO crystal, a tunability of the pump pulse from 370 down to $307 \mathrm{~nm}$ is achieved. Typically $5-7 \mu \mathrm{J} / \mathrm{p}$ are produced in this UV range by mixing $50 \mu \mathrm{J}$ of the fundamental beam with 
about $15-18 \mu \mathrm{J} / \mathrm{p}$ of the visible pulse. The pulse bandwidth achieved in the UV range is around $150 \mathrm{~cm}^{-1}(\simeq 2$ $\mathrm{nm}$ ), which correspond to a FWHM $\sim 70$ fs. However, no compression is used to pre-compensate the chirp introduced by the different optics seen by the UV pump pulse before the interaction region (mostly the BBO crystal, lenses and the vacuum viewport) leading to a longer pulse duration. As probe pulse, either the fundamental pulse $(50 \mu \mathrm{J} / \mathrm{p})$ ), its second harmonic (SHG-15 $\mu \mathrm{J} / \mathrm{p})$ or its third harmonic (THG-6 $\mu \mathrm{J} /$ pulse) is used. The Fourier limited durations expected for these probe pulses from their bandwidths are around 65 fs. The pump and the probe beams are focused in the molecular beam by 250 $\mathrm{mm}$ and $500 \mathrm{~mm}$ lenses, respectively, and combined with a 7 degrees angle between them.

The TTF molecules (Aldrich, $97 \%$ without further purification) are sublimed continuously at $380 \mathrm{~K}$ with 200 Torr of Argon and then expanded through a $250 \mu \mathrm{m}$ nozzle at $450 \mathrm{~K}$ to avoid cork formation. The skimmed molecular beam intersects the focused laser beams perpendicularly, yielding ions which are then detected at the output of an off-axial $39 \mathrm{~cm}$ long time-of-flight velocity-map imaging (VMI) spectrometer. The VMI spectrometer was described previously. ${ }^{33}$ In most of the experiments presented here the polarization of both laser beams are set parallel to each other and parallel to the detector used to collect the photoions or photoelectrons. The imaging detector consists of two microchannel plates (MCPs) coupled to a phosphor screen and a 12-bit CCD camera. The transient ion signals are recorded by using a gated boxcar integrator that collects the amplified output voltage from the phosphor screen coupled by a $100 \mathrm{pF}$ capacitor. For each pump-probe delay, an average over $6 \times 10^{3}$ laser pulses are used by averaging six scans of the delay. A typical photoelectron image was obtained by accumulating around $2 \times 10^{5}$ laser shots and by subtracting the background images of the pump and the probe alone. The corrected images were then Abel inverted to reconstruct the 3D photoelectron distributions, and thus to extract their kinetic energy and angular distributions. The calibration of the photoelectron energy scale was performed by using photoelectron spectra obtained from Xe pumpprobe photoionization. Typically, an energy resolution of $160 \mathrm{meV}$ was obtained at $2 \mathrm{eV}$ kinetic energy by using a repeller plate voltage of $4 \mathrm{kV}$. Note that any energy above $2.2 \mathrm{eV}$, the maximum kinetic energy allowed by the detector size, won't be detected. The energy distribution of photoelectrons are smoothed by a adjacent averaging of four points.

The cross-correlation between the pump and the THG probe is recorded through a $\left(1^{\prime}+1\right)$ photon ionization of $\mathrm{N}, \mathrm{N}$-Dimethylaniline (DMA) resonant at $265 \mathrm{~nm}$ with a low 3s Rydberg state with a long lifetime. ${ }^{34,35}$ The difference between the ionization potential and the onset of the band is indeed around $2.8 \mathrm{eV}$, a gap easily exceeded by the frequency-mixing generated pulse. This ion transient allows to determine precisely the time-overlap between the pump and probe pulses. The cross-correlation time
TABLE I. The cutoff energies of the photoelectrons at $\mathrm{E}_{p h}=$ $\left(\lambda_{\text {pump }}=317 \mathrm{~nm}+\mathrm{n}\right.$ '), or p or p' excitation scheme based on vertical ionization potentials $\mathrm{IP}_{1}=6.7 \mathrm{eV}$ and $\mathrm{IP}_{2}=8.58 \mathrm{eV}$. In pump-probe, due to harmonicity relationship between the different probe wavelengths, the configuration encountered at $800 \mathrm{~nm}$ will be a combination of the $398 \mathrm{~nm}$ and $267 \mathrm{~nm}$ ones. Note that the maximum kinetic energy detectable is $2.2 \mathrm{eV}$.

\begin{tabular}{lccc}
\hline$\lambda_{\text {probe }}[\mathrm{nm}]$ & Excitation & $\mathrm{E}_{p h}-\mathrm{IP}_{1}[\mathrm{eV}]$ & $\mathrm{E}_{p h}-\mathrm{IP}_{2}[\mathrm{eV}]$ \\
\hline 267 & $\left(1+1^{\prime}\right)$ & 1.85 & 0 \\
398 & $\left(1+1^{\prime}\right)$ & 0.32 & not reached \\
398 & $\left(1+2^{\prime}\right)$ & 3.44 & 1.56 \\
\hline$\lambda[\mathrm{nm}]$ & & & \\
317 & 2 & 1.12 & not reached \\
267 & 2 & 2.58 & 0.71 \\
398 & 3 & 2.58 & 0.71 \\
800 & 5 & 1.0495 & not reached \\
\hline
\end{tabular}

extracted by fitting the step function of the DMA cation is $320 \pm 20$ fs as shown in Figure 2. This reveals the strong positive chirp expected for both UV pulses. The cross-correlation time between the pump and the SHG is deduced from the TTF signal itself, as discussed further, while the ones between the pump and the fundamental as probe pulse is measured through the $\mathrm{TTF}_{2}^{+}$signal. Typical cross-correlation times for these two last excitation schemes are slightly reduced to around $300 \mathrm{fs}$.

\section{RESULTS}

In the present experiment, we investigate the excitation region spanning from 322 to $307 \mathrm{~nm}$, in order to reveal a possible change of the electronic character via a variation of the measured decay time or the ionization probability while going through the double absorption feature. The data will be presented by separating them according to the probe pulse wavelength used (267, 398 and $800 \mathrm{~nm}$ ). The transient ion signals recorded at each wavelength combination are fitted by assuming a resonant decay component $\Psi_{d}(t)$ and a non-resonant contribution inherent to this type of experiment $\Psi_{c c}(t)$, corresponding to a cross-correlation signal:

$$
S_{\text {ion }}(t)=y_{0}+\Psi_{c c}(t)+\Psi_{d}(t)
$$

where $y_{0}$ is a background signal. $\Psi_{c c}(t)$ and $\Psi_{d}(t)$ are given by

$$
\begin{aligned}
\Psi_{c c}(t) & =b \times e^{-4 \ln 2 \frac{\tau^{2}}{\tau_{c c}}} \\
\Psi_{d}(t) & =c \times e^{-\frac{\tau}{\tau_{d}}} \times\left[1+\operatorname{erf}\left(\phi\left(\tau, \tau_{d}\right)\right)\right]
\end{aligned}
$$

where the component in the error function is expressed by:

$$
\phi\left(\tau, \tau_{d}\right)=\frac{2 \sqrt{\ln 2}}{\tau_{c c}} \times\left(\tau-\frac{\tau_{c c}^{2}}{8 \ln 2 \times \tau_{d}}\right)
$$


This allows the extraction of the decay times $\tau_{d}$ summarized in Table III, based on the determination of the cross-correlation time $\tau_{c c}$ and the time overlap $\tau=0$.

As expected from the electron donor propriety of this compound, the two first vertical ionization thresholds lie quite low at $\mathrm{IP}_{1}=6.7 \pm 0.02 \mathrm{eV}$ and $\mathrm{IP}_{2}=8.58 \pm$ $0.02 \mathrm{eV} .{ }^{28}$ Within the Koopman's approximation, they correspond mainly to the departure of an electron from the HOMO with a symmetry $b_{3 u}$ and the next channel HOMO-1 with a symmetry $b_{2 g}$. The seven singlet excited states calculated below $4 \mathrm{eV}$ can all be described at more than $80 \%$ by singly electronic excitations. ${ }^{22} \mathrm{All}$ of them are single electron transitions from the HOMO to higher orbitals, except the $1^{1} B_{2 g}$ optically forbidden state with a principal configuration made of $53 \%$ electron removal from the HOMO and $22 \%$ from the HOMO1. This means that photoionization is expected to take place mostly onto the ground state of the cation. Only the $1^{1} B_{2 g}$ state due to its $22 \%$ HOMO- 1 contribution and the Rydberg states due to their peculiar strong propensity for $\Delta \mathrm{v}=0$ ionization, are expected to be set apart from the other contributions in the kinetic energy distribution of the photoelectrons. ${ }^{33,36}$

The excitation schemes for all used wavelength combinations are summarized in Figure 1. One THG photon $(267 \mathrm{~nm})$ is enough to probe the wave packet dynamics onto the ground state of the ion or at the threshold of the first excited state of the cation. On the contrary, when photoionization takes place with a SHG probe $(398 \mathrm{~nm})$, only the ground state of the cation is reached at one photon. With a $800 \mathrm{~nm}$ probe pulse, multiphoton ionization is required to reach any ion continuum. The onset of a higher density of cation states is $\sim 9.5 \mathrm{eV} .^{28}$ Table I summarizes the maximum kinetic energies expected for the photoelectrons via these different ionization schemes. In the following, we will discuss the experimental results, starting with the easiest probe photoionization, namely at one photon (THG probe) and finishing with the most delicate one which is the multiphoton ionization at the fundamental $800 \mathrm{~nm}$ probe pulse.

\section{A. A probe pulse centered at $267 \mathrm{~nm}$}

Figure 2a displays the pump-probe signals of the parent $\mathrm{TTF}^{+}$cations recorded at different pump wavelengths. At a $1+1^{\prime}$ total energy of $\sim 8.6 \mathrm{eV}$, the mass spectra is a single peak of the parent cation. There is no signal at negative delay, even if the $267 \mathrm{~nm}$ probe pulse is on the blue wing of the $4 \mathrm{eV}$ resonance. Underneath, in Figure $2 \mathrm{~b}$, the typical transient of $\mathrm{DMA}^{+}$signal, through which the time overlap $\tau=0$ and the cross correlation time $\tau_{c c}$ are determined, and and $\mathrm{C}_{6} \mathrm{~S}_{4} \mathrm{H}_{4}^{+}$transient are shown. The main result from these $\mathrm{C}_{6} \mathrm{~S}_{4} \mathrm{H}_{4}^{+}$transient study is the invariance of the decay time as a function of the pump wavelength with a typical time constant of $437 \pm 7$ fs. This invariance could suggest that only one electronic state defines the absorption band around $4 \mathrm{eV}$, in contrast to the calculations from Andreu et al. ${ }^{23}$ but in agreement with the ones done by Pou-Armengo et al. using the $\mathrm{D}_{2 h}$ geometry. ${ }^{21}$ The 437 fs decay time could be then assigned to a vibronic coupling from the $1^{1} \mathrm{~B}_{1 u}$ state onto lower electronic states. This conclusion is however too hasty. This long decay is quite unexpected taking into account the large bandwidth, characterizing this absorption band. Within the assumption of a homogeneous broadening due to pure electronic relaxation, the $437 \mathrm{fs}$ decay would correspond to a bandwidth of around 12 $\mathrm{cm}^{-1}$, namely a hundred times narrower than the bandwidths observed on Figure 1. One possible explanation to this counterintuitive invariant decay is that the ion transients reflect a vibrational relaxation. However, the $\sim 500 \mathrm{~cm}^{-1}$ of vibrational energy that we can expect in the $1^{1} \mathrm{~B}_{1 u}$ state is most likely not large enough to reach an intramolecular vibrational relaxation (IVR) threshold of this state, although this compound has 36 normal vibrational modes with strong in-plane force field coupling. Our hypothesis is that the $437 \mathrm{fs}$ decay is the signature of IVR taking place in the electronic state populated by the first internal conversion (IC1) from the zeroth order state(s) (at least $1^{1} \mathrm{~B}_{1 u}$ ) prepared by the pump pulse.

An IC rate is limited by the difference in entropy between the initial and the final electronic states. ${ }^{36}$ When the entropy in the prepared initial state (namely $1^{1} \mathrm{~B}_{1 u}$ ) increases with the same rate as the entropy in the final state (to be determined), the IC rate can reach a converging limit (corresponding to a 437 fs decay) and won't vary anymore as function of the pump energy as observed over the $\sim 200 \mathrm{meV}$ energy region investigated in Figure 2a. ${ }^{36}$ Within this IVR interpretation, the invariance of the decay time observed in Figure 2a would mean only that the electronic state populated by IC1 is the same whatever the pump pulse, but not if one or two electronic states are prepared by the pump pulse.

This hypothesis is confirmed by the photoelectron spectra which are featureless with only the integrated photoelectron intensity decaying as a function of the delay time without large spectral decay. A rotation of the probe polarisation relative to the pump one does not yield to noticeable changes on the photoelectron image apart of a lower total intensity. Whatever the delay, the photoelectrons are mainly produced with a minimum of kinetic energy. This type of energy distribution is quite an issue in time-resolved imaging due to the saturation of the MCP detectors for $\mathrm{E}_{k i n}=0 \mathrm{eV}$ because everything is focused on only on few microchannels. This prevents a proper time-resolution of the photoelectron imaging at that energy. Two photoelectron distributions are displayed in Figure 3 to enlighten the pump-probe ionization process onto the $\mathrm{IP}_{1}$ continuum. At $100 \mathrm{fs}$, when the population is expected to have still the 'zeroth order state' character prepared by the pump pulse, the photoionization is characterized by an ion with at least $\sim 1.3 \mathrm{eV}$ of internal vibrational energy $\left(\mathrm{E}_{k i n}\left(e^{-} \sim 0.5\right)\right.$ $\mathrm{eV})$. As soon as $200 \mathrm{fs}$ (here shown at $400 \mathrm{fs}$ ) this maximal internal energy is reduced by half $\left(\mathrm{E}_{k i n}\left(e^{-} \sim 1.1\right)\right.$ 


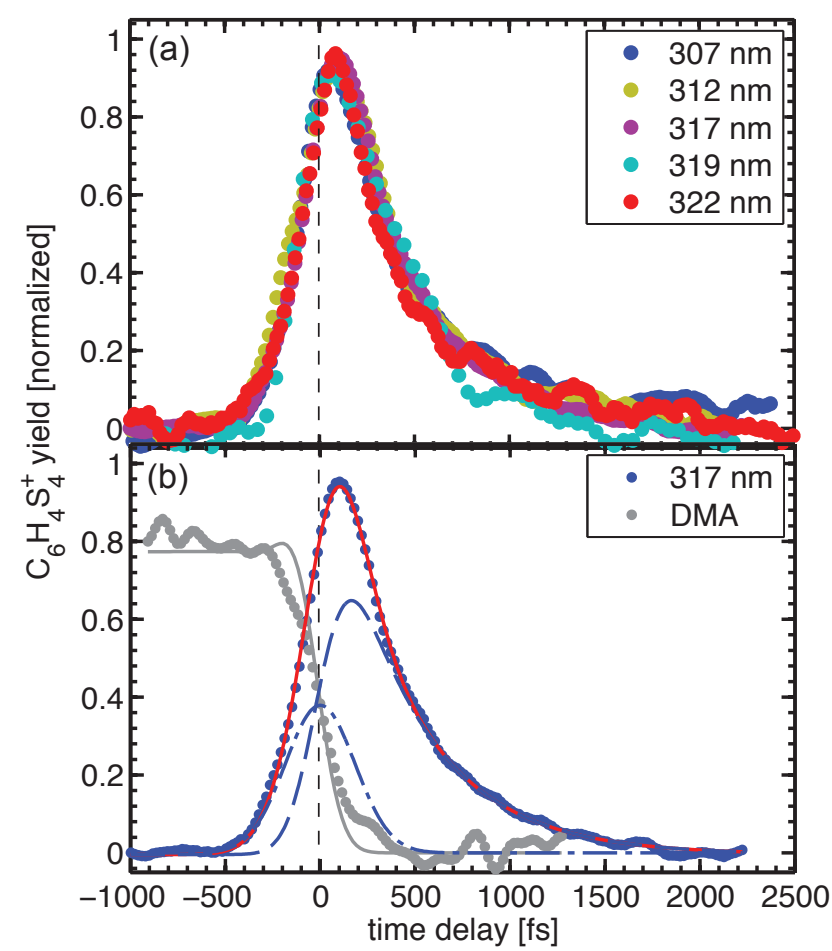

FIG. 2. Pump-probe time transients with a probe centered at $267 \mathrm{~nm}$. (a) Femtosecond time-resolved transients of $\mathrm{TTF}^{+}$as function of the pump pulse wavelength. (b) N,NDimethylaniline transient at $267+317 \mathrm{~nm}$ that produces a long lifetime transient through a 3s-Rydberg excitation (stepfunction) from which the time overlap $(\tau=0 \mathrm{fs})$ and the cross correlation time of this UV-UV pump-probe excitation are both extracted. The $T T F^{+}$time transient (shown at $317 \mathrm{~nm}$ full dot) can be fitted (red) by the sum of a crosscorrelation component $\left(\Psi_{c c}(t)\right.$, dash-dot) and a decay component $\left(\Psi_{d}(t)\right.$,dashed $)$.

$\mathrm{eV})$. Afterward the photoelectron energy distribution changes only in intensity. This is in contrast to most time-resolved photoelectron spectroscopy studies done in large systems. In general, the photoelectron kinetic energy decreases due to the rising of the vertical ionization potential when vibrational energy increases in the neutral molecule. Our observations can be rationalized if the equilibrium geometry of the electronic excited state prepared by the pump pulse is shifted relative to the cation one and if the electronic state populated by the IC1 has a equilibrium-like geometry than the cation one. In that case, smaller internal energies of the cation would be produced once dynamics take place within the IC1 populated electronic state. In that context, it is important to remember that although computational methods failed by $0.6 \mathrm{eV}$ to reproduce the experimental vertical ionization potential IP1, they predict at the optimized geometry of the neutral ground state, a $200 \mathrm{meV}$ energy difference between the adiabatic and vertical ionization potentials. ${ }^{21}$

Similar findings are observed for the one color two- photon ionizations with the pump or probe beam alone, as shown in Figure 3b. This fs-multiphoton transition should reflect ionization taking place from the vibrationally cold ground electronic state with a limiting effect from intermediate resonances. Namely, the clear energy cut-off of the IP1 threshold should appear in the kinetic energy distribution of the photoelectrons, as already observed at $800 \mathrm{~nm}$ with a 80 fs pulse. ${ }^{29}$ At $2 \times 317$ $\mathrm{nm}$, with only $\mathrm{IP}_{1}$ accessed, the ionization probability is almost totally flat over the total $1.1 \mathrm{eV}$ of vibrational energy available. A similar conclusion is reached at 2 $\times 267 \mathrm{~nm}$, although the complete vibrational window can not be accessed in this case due to the detector size. Clearly, the intermediate resonance around $4 \mathrm{eV}$ affects drastically the Franck-Condon window for a $\sim 200$ fs chirped UV pulse.

Since the flat distribution observed at a time delay of 400 fs does not fully occupy the opened vibrational window (i.e. $1.1 \mathrm{eV}$ observed, instead of the $1.85 \mathrm{eV}$ opened in the $\mathrm{IP}_{1}$ continua at $\left.1+1^{\prime}\right)$, we could conclude that at this time delay, not all of the vibrational population has been detected via this $1+1$ ' ionization. Within the current interpretation, IVR is expected to change the Franck-Condon overlap in the photoionization such that almost the entire energy range is used in the $\mathrm{IP}_{1}$ continuum, but not completely, with a higher probability to produce ions with small internal energy at 400 fs than at $100 \mathrm{fs}$. The remaining question is what the involved IC1 electronic state is. Calculations in MS-CASPT2 in the $\mathrm{D}_{2 h}$ geometry predict four triplet states and seven singlet states below $4 \mathrm{eV}$, including two Rydberg states, one $3 \mathrm{~s}$ state at $3.6 \mathrm{eV}$ and one $3 \mathrm{p}_{z}$ state at $3.8 \mathrm{eV} .^{21}$

The angular distributions of the photoelectron images have been analyzed in two energy ranges from 0.11 to 0.36 $\mathrm{eV}$ and from 0.36 to $1.2 \mathrm{eV}$. With the laser polarization being linear and parallel to each other and the detector, the angular distribution is defined by a series of even Legendre polynomials up to the second order for the $1+1$ ' photoionization:

$$
I(\theta)=1+\sum_{n=2} \beta_{2 n} P_{2 n}(\cos \theta)
$$

with $P_{2}(x)=\frac{1}{2}\left(3 x^{2}-1\right)$ and $P_{4}(x)=\frac{1}{8}\left(35 x^{4}-30 x^{2}+3\right)$. Table 2 summarizes the coefficients obtained for the four images of Figure 3.

By comparing the one color $\beta$-parameters recorded at $317 \mathrm{~nm}$ and $267 \mathrm{~nm}$, we see that the resonance encountered at $4 \mathrm{eV}$ reduces the anisotropy of the photoelectron emission regardless of the photoelectron kinetic energy. Indeed the $\beta_{2}$ parameters for $317 \mathrm{~nm}$ are about half the magnitude of those at $267 \mathrm{~nm}$ with minor contribution for $\beta_{4}$ at resonance. For a well established resonance, namely the pump-probe images, anisotropy is more important at $\tau=100 \mathrm{fs}$ than at $400 \mathrm{fs}$, being almost isotropic for all excitation energies at this delay with no signature of the two-photon ionization $\left(1+1^{\prime}\right)$, as revealed by $\beta_{4} \sim 0$ contribution. 
TABLE II. Anisotropy parameters for the photoelectron images shown in Fig. 3 and 5. The low energy range (LE) corresponds to photoelectron with kinetic energy between 0.11 to $0.36 \mathrm{eV}$, while the high energy (HE) range from 0.36 to 1.2 $\mathrm{eV}$.

\begin{tabular}{lcccc}
\hline$\lambda /$ Delay & & $\beta_{2}$ & $\beta_{4}$ & $\beta_{6}$ \\
\hline $317 \mathrm{~nm}$ & LE & $0.14 \pm 0.01$ & $-0.056 \pm 0.007$ & \\
& HE & $0.39 \pm 0.01$ & -0.003 & \\
\hline $267 \mathrm{~nm}$ & LE & $0.24 \pm 0.01$ & $0.03 \pm 0.01$ & \\
& HE & $0.72 \pm 0.02$ & $0.19 \pm 0.02$ & \\
$100 \mathrm{fs}$ & LE & $0.32 \pm 0.01$ & $0.12 \pm 0.01$ & \\
& HE & $1.18 \pm 0.01$ & $0.43 \pm 0.02$ & \\
$400 \mathrm{fs}$ & LE & $0.12 \pm 0.01$ & $-0.052 \pm 0.006$ & \\
& HE & $0.42 \pm 0.01$ & $0 \pm 0.003$ & \\
\hline $398 \mathrm{~nm}$ & & & & \\
$150 \mathrm{fs}$ & LE & $0.23 \pm 0.01$ & $-0.105 \pm 0.01$ & \\
& HE & $0.74 \pm 0.02$ & $-0.06 \pm 0.02$ & $0.25 \pm 0.03$ \\
$400 \mathrm{fs}$ & LE & $0.39 \pm 0.02$ & $-0.076 \pm 0.015$ & \\
& HE & $1.22 \pm 0.03$ & $0.15 \pm 0.04$ & $0.45 \pm 0.04$ \\
\hline
\end{tabular}

Note that the $3 \mathrm{~s}$ and $3 \mathrm{p}_{z}$ Rydberg states converge onto the ground state of the cation. ${ }^{21}$ If a $3 \mathrm{p}_{z}$ Rydberg state, with $\sim 100 \mathrm{meV}$ of vibrational energy at $317 \mathrm{~nm}$, would be populated directly or by IC1, a low anisotropy at a well-defined kinetic energy around $1.7 \mathrm{eV}$ of the photoelectron would be expected, due to the interferences between the s and d outgoing waves and the conservation of vibrational energy in the ionization process respectively. The lack of a Rydberg fingerprint in the featureless energy distribution of the photoelectron spectrum let us discard a pure $3 \mathrm{p}_{z}$ Rydberg state as a IC1 electronic state. $^{37}$ For the 3s Rydberg state with $\sim 300 \mathrm{meV}$ of vibrational energy at $317 \mathrm{~nm}$, a strong anisotropy due to the pure $\mathrm{p}$ outgoing wave $\left(\beta_{2}=2\right)$ would be expected at a kinetic energy around $1.6 \mathrm{eV}$. The decrease of anisotropy as a function of time as well as the lack of a clear component around $1.6 \mathrm{eV}$ discards as well the implication of the 3s Rydberg state.

In the next two investigations, the probe wavelength is changed (to 398 and $800 \mathrm{~nm}$ ) to confirm the long lifetime measurement and as well to enlighten the states involved in the relaxation process. The pump wavelength is kept around $317 \mathrm{~nm}$.

\section{B. A probe pulse centered at $398 \mathrm{~nm}$}

A probe pulse centered at $398 \mathrm{~nm}$ has the main advantage to be off resonance at 1 photon, while the $1+2$ ' excitation scheme will open properly the second ionization continuum to $\mathrm{IP}_{2}$. Indeed, a $200 \mathrm{~nm}$ probe would have produce a too large background taking the high excitation cross-section at that wavelength.

The $\mathrm{TTF}^{+}$transient recorded with $\lambda_{\text {probe }}=398 \mathrm{~nm}$, shown on Figure 4, has a typical decay time of $367 \pm$ $11 \mathrm{fs}$ which is about $16 \%$ lower than the previous one

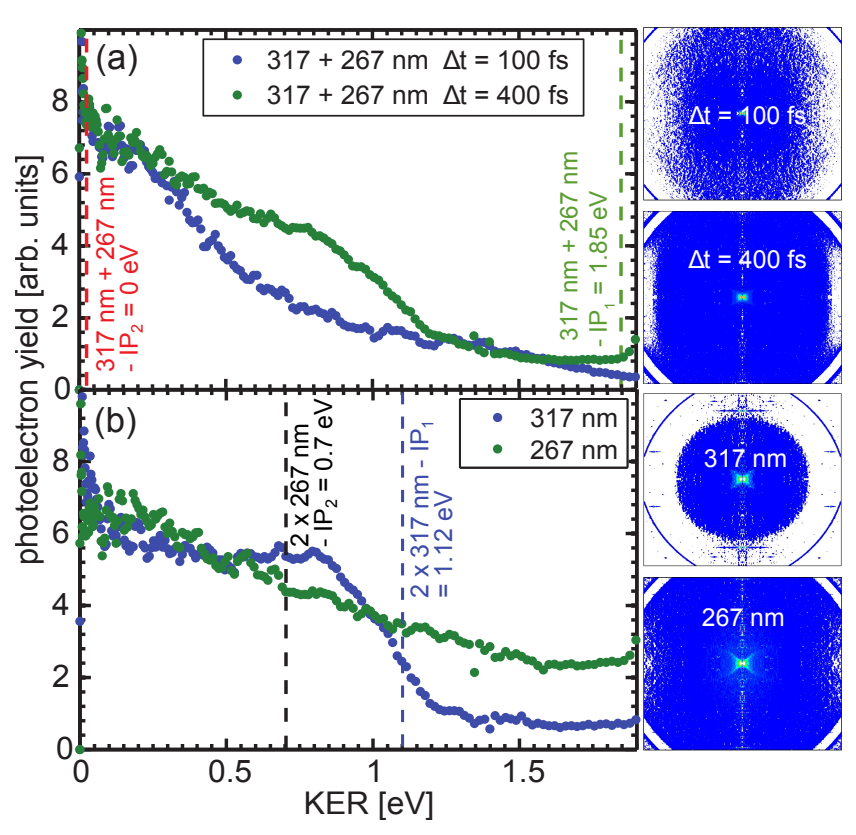

FIG. 3. Photoelectron energy distribution at $317+267 \mathrm{~nm}$ (background free). Similar photoelectron spectra have been observed at the other pump wavelengths between 322 and 307 $\mathrm{nm}$. (a) as a function of the pump-probe time delay (b) the pump and the probe alone. The images on the side are $10^{5}$ laser shot acquisitions and background free. The vertical reference lines are respectively: $317 \mathrm{~nm}+267 \mathrm{~nm}-\mathrm{IP}_{1}$ (green), $317 \mathrm{~nm}+267 \mathrm{~nm}-\mathrm{IP}_{2}$ (red), $2 \times 317 \mathrm{~nm}-\mathrm{IP}_{1}$ (blue) and $2 \times 267 \mathrm{~nm}-\mathrm{IP}_{2}$ (black). The limit for $267 \mathrm{~nm}-\mathrm{IP}_{1}$ is at 2.55 $\mathrm{eV}$, beyond the detector limit.

with a probe beam at $267 \mathrm{~nm}$. The photoelectron spectra, shown in Figure 5, reveal that photoionization taking place at $\left(1+1^{\prime}\right)$ energy- $7 \mathrm{eV}$-only $300 \mathrm{meV}$ above the $\mathrm{IP}_{1}$ threshold is strongly shifting from $200 \mathrm{meV}$ to $0 \mathrm{eV}$ during the first $400 \mathrm{fs}$. A similar effect is as well observed for the $1+2$ ' channel. For time delays of $150 \mathrm{fs}$ it is worthy to note that the energy distribution is in agreement with the energy cutoff expected for the second continuum of ionization IP2 $(1.56 \mathrm{eV})$. However, within the Koopman's approximation, this is only possible for photoionization taking place from an excited electronic state with a $b_{3 g}$ hole. There are two possible ways to get such photoionization spectrum. At one photon of the pump, this would mean that the $1^{1} B_{2 g}$ state (calculated at 3.41 $\mathrm{eV}^{21}$ ) is populated by IC1 from the 'zeroth order' electronic state(s that includes $\left.2^{1} B_{1 u}\right)$. At $1+1^{\prime}-7 \mathrm{eV}$ - a Rydberg state converging to $\mathrm{IP}_{2}$ is populated. This latter hypothesis would explain as well why no contribution for $1+2$ '-IP1 is observed. Rydberg states converging to IP2 have been indeed detected at $6 \mathrm{eV} .^{29}$ The two ionization processes $\left(1+1^{\prime}\right)$ and $\left(1+2^{\prime}\right)$ will be discussed independently.

In the $\left(1+1^{\prime}\right)$ channel, photoelectrons appear with a kinetic energy $\sim 200 \mathrm{meV}$ during the first 300 fs before to be reduced. A transient from a $3 \mathrm{p}_{z}$ Rydberg state would be expected at $200 \mathrm{meV}$. The angular distribution of this 


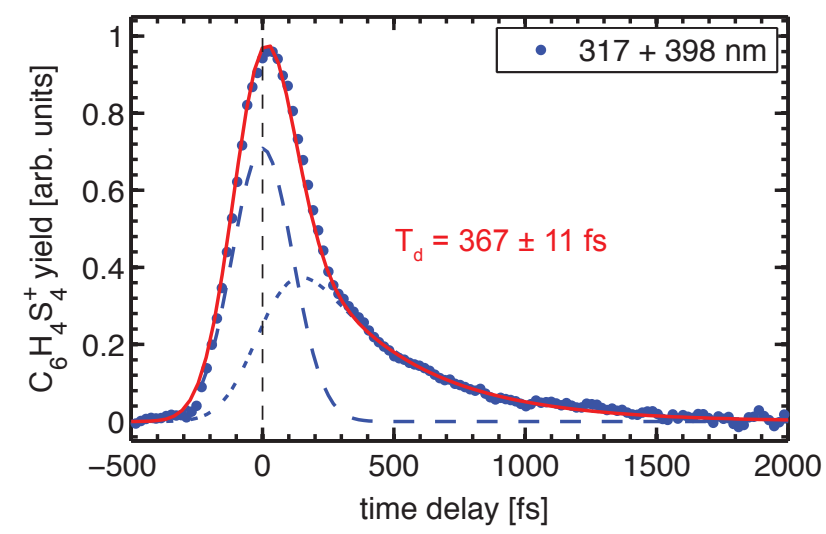

FIG. 4. Time transient of the parent molecule TTF recorded with a probe pulse at $398 \mathrm{~nm}$.

low energy range is quite isotropic (see Table II), consistent also with a $3 \mathrm{p}_{z}$ Rydberg state. However, there is no obvious reason why a Rydberg fingerprint for that state will appear with $\lambda_{\text {probe }}=398 \mathrm{~nm}$ and not with $\lambda_{\text {probe }}=$ $267 \mathrm{~nm}$. This is the main reason why we still discard a $3 \mathrm{p}_{z}$ Rydberg assignment.

In the $\left(1+2^{\prime}\right)$ - $\mathrm{IP}_{2} /-\mathrm{IP}_{1}$ channel, the photoelectron energy distribution spreads almost over the entire $1.5 \mathrm{eV}$ vibrational energy window with a higher probability at small kinetic energies as already observed for the $\left(1+1^{\prime}\right)$ channel - IP 1 at $267 \mathrm{~nm}$. The cut-off of IP2 is clearly observed, especially at $150 \mathrm{fs}$ and $300 \mathrm{fs}$. This ionization onto the IP2 continuum is not expected taking into account the singly electronic excitation from the HOMO that defines most of the singlet excited state. As already discussed previously, only the $1^{1} B_{2 g}$ state due to its $22 \%$ HOMO-1 character is correlated to this ion continuum. Our tentative assignment is that the IC1 populates at least this state, that has been calculated at $3.41 \mathrm{eV}{ }^{21}$ The minimal vibrational energy of the cation observed at long time delays in the $\mathrm{IP}_{2}$ continuum by $\left(1+2^{\prime}\right)$ is $400 \mathrm{meV}$, which is in agreement with the $500 \mathrm{meV}$ expected in the $1^{1} B_{2 g}$ with a $317 \mathrm{~nm}$ pump. In that context the 367 fs decay of the ion transient will show similarly to $\left(1+1^{\prime}\right)$ at $267 \mathrm{~nm}$ the IVR taking place onto the the $1^{1} B_{2 g}$ state. Note however, that another possible explanation for the IP2 ionization is as well a Rydberg state populated at $1+1$ ' (around $7 \mathrm{eV}$ ), correlated to the IP2 continuum as already observed. ${ }^{29}$

At high energy (HE) range, the $\beta_{2}$ parameter slightly increases as expected for a (1+2') ionization compared to a $\left(1+1^{\prime}\right)$ one. In order to take into account the three photons involved in the $(1+2$ ') scheme, the angular fit includes a $\beta_{6}$ contribution for the $\mathrm{HE}$ range. On the contrary to $\lambda_{\text {probe }}=267 \mathrm{~nm}$, the anisotropy of this $\mathrm{HE}$ range, slightly increases at longer delay. No signature of the two Rydberg states lying around $3.6 \mathrm{eV}$ can be expected in this $\left(1+2^{\prime}\right)$ HE range since both converged to the ground state of the cation and the kinetic energy window is limited to $2.2 \mathrm{eV}$.

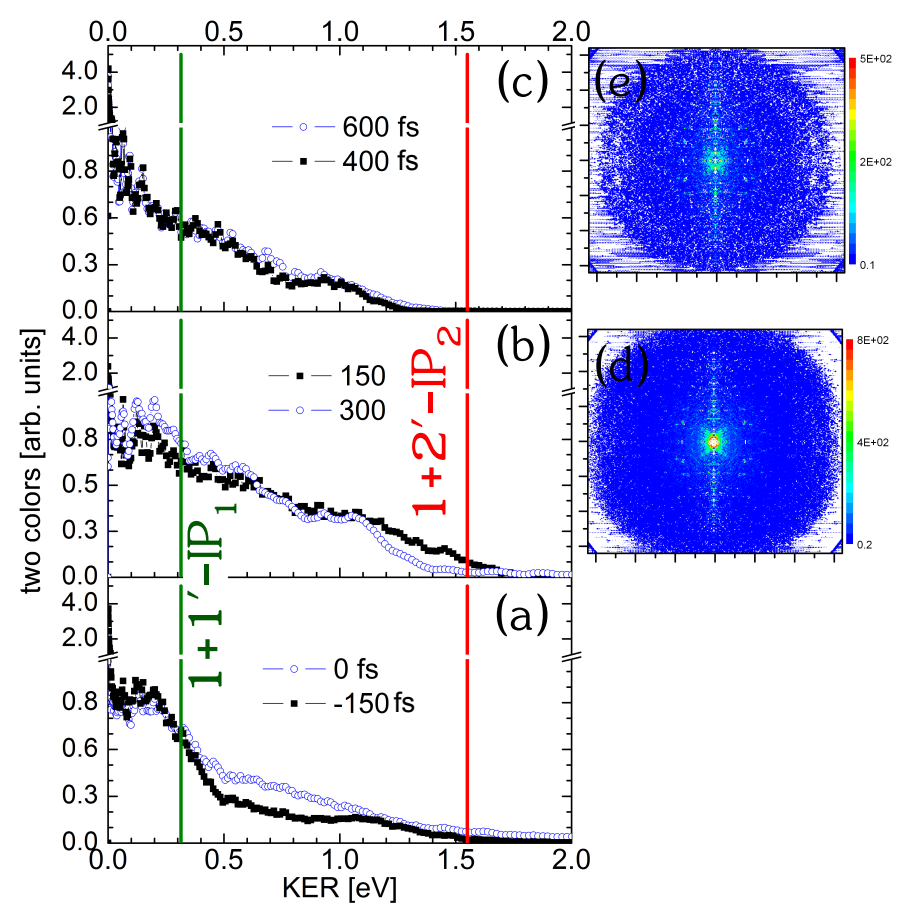

FIG. 5. Photoelectron energy distribution for different pumpprobe delay and $\lambda_{\text {probe }}=398 \mathrm{~nm}(\mathrm{a})-150$ and $0 \mathrm{fs}$ (b) 150 and $300 \mathrm{fs}$ (c) 400 and $600 \mathrm{fs}$. The image (d) and (e) corresponds to the 150 and 400 fs delays respectively. Same acquisition time than Figure 3. The vertical reference lines are respectively : $318 \mathrm{~nm}+398 \mathrm{~nm}-\mathrm{IP}_{1}$ (green), $318 \mathrm{~nm}+2 \times 398 \mathrm{~nm}-\mathrm{IP}_{2}$ (red)

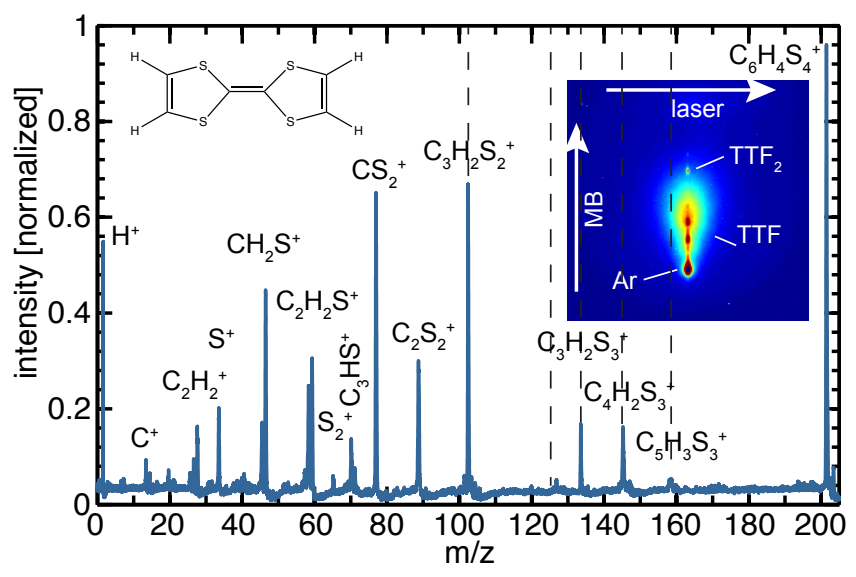

FIG. 6. Mass spectrum recorded at +200 fs between a pump pulse centered at $318 \mathrm{~nm}$ and an intense probe pulse centered at $800 \mathrm{~nm}$. The insert figure is the image of the mass species produced in the continuous molecular beam at $\tau=0$ fs.

\section{A probe pulse centered at $800 \mathrm{~nm}$}

As we will see, tuning the probe wavelength to $800 \mathrm{~nm}$ with a typical intensity of $50 \mu \mathrm{J} / \mathrm{p}$ reveals a relaxation taking place on two different timescales. The $\mathrm{IP}_{1}$ ionization continuum is reached in a $\left(1+2^{\prime}\right)$ scheme with a small excess energy of $\sim 300 \mathrm{meV}$. This is similar to the 
$\left(1+1^{\prime}\right)$ scheme at $398 \mathrm{~nm}$, seen before. Above threshold ionization (ATI) takes place from both the $\mathrm{IP}_{1}$ and $\mathrm{IP}_{2}$ opening the ion continuum up to $15 \mathrm{eV}$ as already studied in a similar experimental conditions with a larger detector to access higher photoelectron energies. ${ }^{29}$ In a VUV one-photon ionization, dissociative ionization onto the largest primary fragments is complete as low as 10 eV. ${ }^{29}$ Figure 6 shows the mass spectrum recorded at $\tau$ $=200 \mathrm{fs}$. Dissociative ionization clearly dominates up to the formation of sulfur cations. In the VUV coincidence experiment, ${ }^{29}$ the $\mathrm{S}^{+}$fragment is produced by a secondary dissociation of $\mathrm{S}_{2}$, formed after large rearrangement of $\mathrm{TTF}^{+}$with an appearance energy of 12.6 $\mathrm{eV}$. This mass spectrum is quite similar to the helium collision-induced dissociation mass spectrum of TTF. ${ }^{38}$ The $\tau=0$ fs ion image shown in Figure 6 reveals not only the main contribution from TTF but more surprising the formation of the dimer of TTF in this continuous molecular beam. There is no spectroscopic data published on this dimer.

The time dependencies of the main species are shown on Figure 7. The time dependency of the dimer shows clearly a shoulder and a depleted background signal at positive delay. This is a signature of a resonance encountered at $317 \mathrm{~nm}$ in these dimers. The cross-correlation time as well as the time overlap is determined by fitting the negative delay part of this dimer transient. $\tau_{c c}$ is measured to be $295 \pm 7$ fs and the decay time of $\mathrm{TTF}_{2}^{+}$ extracted from this resonant transient is $134 \pm 20 \mathrm{fs}$.

All decay times measured for the different masses are summarized in Table III and do not depend on the probe intensity which was varied between 30 and $90 \mu \mathrm{J} / \mathrm{p}$. The main result is that the decay time on the $\mathrm{TTF}^{+}$signal is $40 \%$ shorter than the ones measured before with a probe pulse centered at $267 \mathrm{~nm}$ or $398 \mathrm{~nm}$. We assign this 260 fs decay to IC1 which is unrevealed in the ion transients with UV probes. The higher the appearance energy of the fragment produced by dissociative ionization, the longer is the decay time. It is as well a non-decaying step appearing in the same manner. In order to check that the dynamics are still initiated at $317 \mathrm{~nm}$, a pump power dependency of $\mathrm{C}_{2} \mathrm{H}_{2} \mathrm{~S}_{2}^{+}$and $\mathrm{S}^{+}$is measured at $\tau=4 \mathrm{ps}$ which is shown in the inserts of Figure 7 for these fragments. The linear dependency observed for both fragments confirms that the dynamics are indeed initiated at $3.9 \mathrm{eV}$ and no dissociation takes place from multiphoton absorption of the pump pulse, as already observed in other systems. ${ }^{39}$ The presence of a non-decaying step at more than 6 ps can be assigned to a second internal conversion IC2 onto the ground state. In order to take into account this new contribution, an independent rising component with a typical time constant $\tau_{r}$ is added to equation 1 :

$$
\Psi_{r}(\tau) \propto\left(1-e^{-\frac{\tau}{\tau_{r}}}\right) \times\left[1+\operatorname{erf}\left(\phi\left(\tau, \tau_{r}\right)\right)\right]
$$

The fits converge systematically to $\tau_{d}=\tau_{r}$. This means that both the electronically excited state populated by

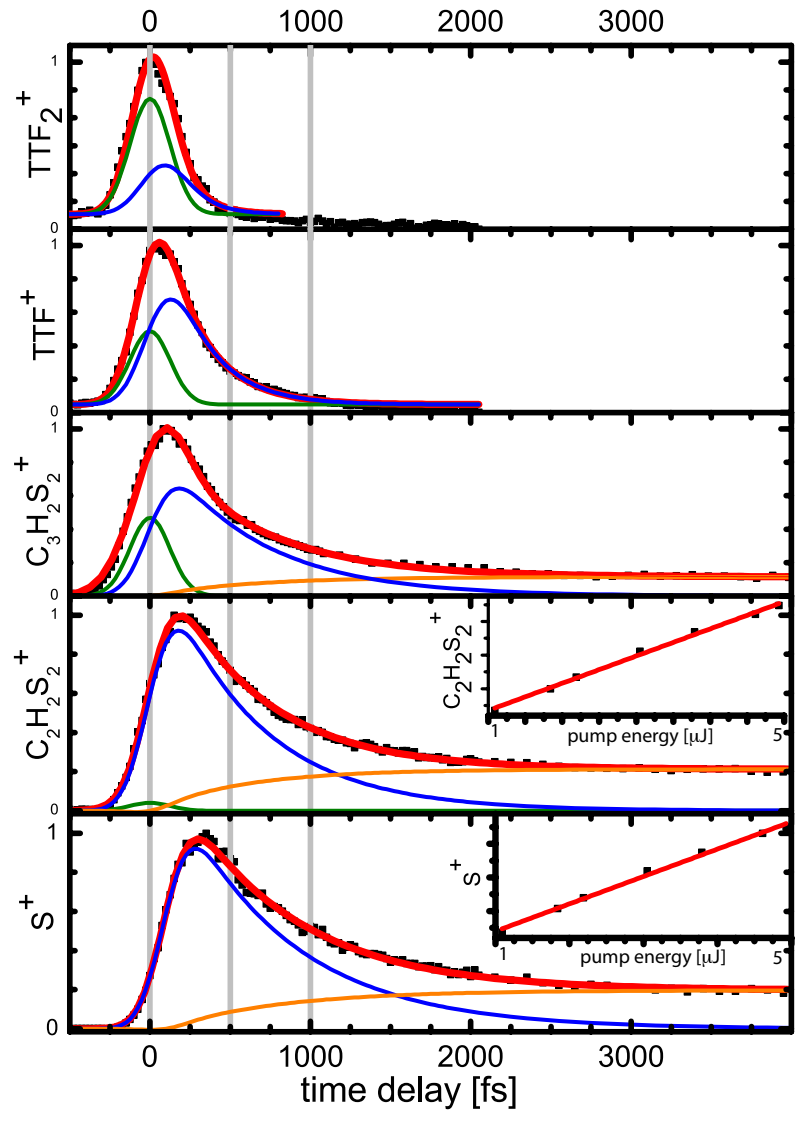

FIG. 7. Time transients of different cations with a probe pulse centered at $800 \mathrm{~nm}$. The insert graphs show the pump power dependency of the fragments at delay 4 ps.

IC1 and the ground electronic state have their dynamics projected onto the dissociative ionization continuum, when this later is energetically allowed, but with a lower probability for the ground electronic state.

These observations are similar to the ones obtained, for instance, on $\mathrm{Cr}(\mathrm{CO})_{6}$ or norbornene probed with an intense probe pulse. ${ }^{40,41}$ Here as well the degree of fragmentation is weaker at small pump-probe delays and the decay time increases as the mass of the fragments is decreasing. Indeed, when the molecule relaxes, the electronic energy is converted into vibrational energy, which upon ionization remains in the ion. This is causing dissociative ionization once its threshold is energetically reached. Below this threshold, as observed for $\lambda_{\text {probe }}=268 \mathrm{~nm}$ or $398 \mathrm{~nm}$, photoionization takes place whatever the nuclear geometry with almost the same probability. This leads to the relative featureless photoelectron spectra observed in Figure 3 and 5. This applies because the energies of the ion bonds are weaker than the binding energies of the neutral molecule. When electronic relaxation onto the ground electronic state takes place, vibrational energy becomes important. Therefore, upon photoionization, the internal energy of the ion will be more important and a fragmentation down to the smaller species will take place. This hypothesis is supported by the largest shift 
TABLE III. The different decay times extracted by the fit procedure based on equation 1, completed with equation 3 for the fragments of dissociative ionization. The cation transients are shown in Figures 2, 4 and 7. The appearance energies of the cation are extracted from ${ }^{29}$. ( ${ }^{*}$ with a delay shift of $95 \pm$ $7 \mathrm{fs})$.

\begin{tabular}{ccccc}
\hline$\lambda_{\text {probe }}[\mathrm{nm}]$ & Ion & $\mathrm{E}_{A}[\mathrm{eV}]$ & $\tau_{d}[\mathrm{fs}]$ & $\tau_{c c}[\mathrm{fs}]$ \\
\hline 267 & $C_{6} H_{4} S_{4}^{+}$ & $6.7\left(\mathrm{IP}_{1}\right)$ & $437 \pm 7$ & $304 \pm 2$ \\
398 & $C_{6} H_{4} S_{4}^{+}$ & - & $397 \pm 11$ & $272 \pm 3$ \\
800 & $C_{6} H_{4} S_{4}^{+}$ & - & $259 \pm 7$ & $295 \pm 7$ \\
800 & $C_{3} H_{2} S_{2}^{+}$ & 11 & $615 \pm 21$ & - \\
800 & $C_{2} H_{2} S_{2}^{+}$ & 10 & $576 \pm 9$ & - \\
800 & $S^{+}$ & 12.6 & $704 \pm 13^{*}$ & - \\
\hline
\end{tabular}

observed on the maximum of $\mathrm{S}^{+}$signal that requires to introduce a real time shift of 95 fs to reproduce its transient. We recall the assumption that fragments coming from the dissociation of neutral molecules, are not involved in this process. This has been checked by monitoring the VMI images of these fragments. They all show tiny kinetic energy releases as expected for dissociative ionization. Dissociative ionization allows to detect the end products of ultrafast relaxation in TTF since it gives rise to a non-vanishing signal of infinite lifetime.

Figure 8 shows the time dependency of the photoelectron produced in such an excitation scheme. Taking into account the dissociative ionization, the different possible ion continua as well as the limitation of observation fixed by the size of the detector, an assignment of the time-dependent peaks is totally irrelevant. Moreover, in systems where multiphoton ionization is involved, doubly excited states can be populated at $\left(1+1^{\prime}\right)$ energy, leading to Rydberg fingerprints in the photoelectron spectra. ${ }^{36,37,42}$

\section{DISCUSSION}

In the following we propose and discuss a relaxation mechanism to rationalize the three time constants observed, namely $\sim 260$ fs on $\mathrm{TTF}^{+}$at $\lambda_{\text {probe }}=800 \mathrm{~nm}, \sim$ $400 \mathrm{fs}$ on $\mathrm{TTF}^{+}$both with $\lambda_{\text {probe }}=267$ and $398 \mathrm{~nm}$ and finally for the fragments the longest timescales which are all $>580$ fs revealed by dissociative ionization. The shortest decay time of $260 \mathrm{fs}$ is too long to be a Franck-Condon window departure and should reflect the first internal conversion IC1 onto a slightly lower electronic state that we tentatively assign to $1^{1} B_{2 g}$ state due to the IP2 continuum opened at $1+2$ ' with $\lambda_{\text {probe }}=398 \mathrm{~nm}$. Rydberg states are discarded due to the absence of a clear vibrational or angular signature on the photoelectron spectra at $\lambda_{\text {probe }}=268 \mathrm{~nm}$. Since the time transients with the UV probe pulses are both fitted by a single exponential function with a decay constant of $\sim 400 \mathrm{fs}$, the electronic state reached by IC1 must be close in energy to the 'zeroth order' state prepared by the pump pulse. This is

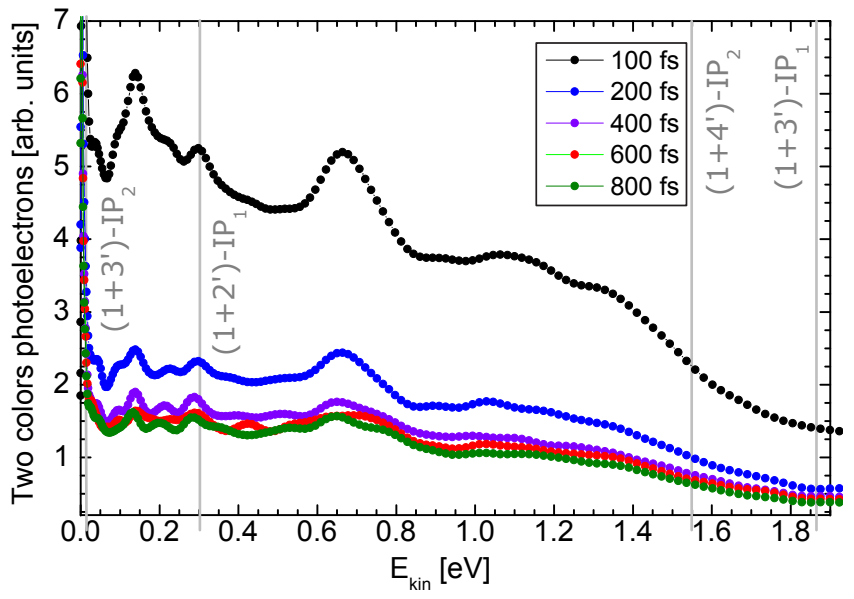

FIG. 8. Photoelectron energy distribution as a function of the pump-probe delay. The vertical reference lines indicate the maximum kinetic energy for the different channels of ionization $\left(1+\mathrm{n}\right.$ ') onto the $\mathrm{IP}_{1}$ and $\mathrm{IP}_{2}$ ionization continuum after the absorption of one photon of the pump pulse and n' photons of the probe pulse.

indeed the case for the $1^{1} B_{2 g}$ state. In addition, this newly populated state should have a similar ionization cross-section than the 'zeroth order state' to not be revealed in the ion transients of Figure 2 and 4 . The $\sim$ 400 fs decay time, revealed in these UV ion transients, reflects then the IVR taking place into the $1^{1} B_{2 g}$ state.

By reaching higher energies in the ion continuum via multiphoton ionization at $800 \mathrm{~nm}$, the transients of the dissociative ionization fragments reveal two components with similar time constants, namely a decaying one and a rising one in the range of $580 \mathrm{fs}$. This would reflect the transfer of population from $1^{1} B_{2 g}$ - the decaying component - to the ground electronic state - the long lifetime step showing the appearance of the end product of this photochemical reaction. This is the simplest story that can be build from the observed transients. To finish, it is quite interesting to notice that the 400 fs decay time measured with the UV probe pulses is never observed with the IR probe pulse on the parent cation, even though the three probe wavelengths are linked by the harmonic relationship. Maybe the intermediate resonance reached at $\left(1+1^{\prime}\right)$ for $\lambda_{\text {probe }}=800 \mathrm{~nm}, 5.45 \mathrm{eV}$, affects the FranckCondon window of ionization.

\section{v. CONCLUSION}

The ultrafast dynamics taking place from the second band, which is optically allowed in TTF, has been revealed by both, varying the pump and the probe wavelengths. The observed time dependencies give no evidence which would indicate the presence of two different electronic states or of Rydberg states in the observed energy range, as suggested in the past. Possibly this double structure could be assigned to a 
vibrational pattern as suggested by Pou-Armengo et al. The ultrafast dynamics in TTF are characterized by two time scales as revealed by changing the probe wavelengths. The first internal conversion seems to be achieved in 260 fs followed by a second one onto the ground electronic state with a slightly longer time constant around 600 fs. Dissociative ionization and UV ionization allow to monitor as well the transfer from electronic to vibrational energy in all the electronic states populated by the internal conversions, namely the $1^{1} B_{2 g}$ state and the ground state. Monitoring similar TTF compounds, ${ }^{3,30}$ might as well confirm the generality of this long electronic and vibrational relaxation. This approach is relevant to optimize the design of new photoactive materials incorporating a TTF core or derivatives. In this gas phase study, the formation of a TTF dimer has been as well revealed.

This work was supported financially by the ANR08-JCJC-0029 HARMODYN, ANR-14-CE32-0014 MISFITS and l'Université Paul Sabatier. D.S. acknowledges the European Union for its PhD fellowship from the ITNICONIC (FP7-Grant Agreement No. 238671). We thank Elsa Baynard, Stéphane Faure and William Volondat for their expert technical assistances.

${ }^{1}$ Fred Wudl, Darold Wobschall, and Earl J Hufnagel. Electrical conductivity by the bis(1,3-dithiole)-bis(1,3-dithiolium) system. J. Am. Chem. Soc., 94(2):670-672, January 1972.

${ }^{2}$ R. Gleiter, E. Schmidt, D. O. Cowan, and J. P. Ferraris. J. Elect. Spect. and Rel. Phen., 2(2):207, 1973.

${ }^{3}$ J. L. Segura and N. Martin. Angewandte Chemie - International Edition, 40(8):1372, 2001.

${ }^{4}$ J. Becher, J. O. Jeppesen, and K. Nielsen. Synthetic Metals, 133-134:309, 2003.

${ }^{5}$ F. Sawano, I. Terasaki, H. Mori, T. Mori, M. Watanabe, N. Ikeda, Y. Nogami, and Y. Noda. Nature, 437(7058):522, 2005.

${ }^{6}$ Chen-Jie Fang, Zhi Zhu, Wei Sun, Chun-Hu Xu, and Chun-Hua Yan. New TTF derivatives: several molecular logic gates based on their switchable fluorescent emissions. New Journal of Chemistry, 31(4):580, 2007.

${ }^{7}$ D Canevet, M Sallé, G Zhang, and D Zhang. Tetrathiafulvalene (TTF) derivatives: key building-blocks for switchable processes. Chem. Comm., 2009.

${ }^{8}$ Chunyang Jia, Jiaqiang Zhang, Jingying Bai, Ligong Zhang, Zhongquan Wan, and Xiaojun Yao. Synthesis, physical properties and self-assembly of conjugated donor-acceptor system based on tetrathiafulvalene and functionalized with binding sites. Dyes and Pigments, 94(3):403-409, September 2012.

${ }^{9}$ Istvan Hargittai, Jon Brunvoll, Maria Kolonits, and Vladimir Khodorkovsky. J. Molec. Struct., 317(3):273, 1994.

${ }^{10}$ Ersan Demiralp and III Goddard, William A. J. Phys. Chem. A, 101(43):8128, 1997.

${ }^{11}$ R. Viruela, P. M. Viruela, E. Orti, and R. Pou-Amerigo. Synthetic Metals, 103(1-3):1991, 1999.
${ }^{12}$ A. Graja, I. Olejniczak, and A. Bogucki. J.of Molec. Struct., 614(1-3):59, 2002.

${ }^{13}$ Nazario Martin, Luis Sanchez, M Angeles Herranz, and Dirk M. Guldi. J. Phys. Chem. A, 104(19):4648-4657, 2000.

${ }^{14}$ D. Kreher, P. Hudhomme, A. Gorgues, H. X. Luo, Y. Araki, and O. Ito. Phys. Chem. Chem. Phys., 5(20):4583-4592, 2003.

${ }^{15} \mathrm{P}$. A. van Hal, R. A. J. Janssen, G. Lanzani, G. Cerullo, M. Zavelani-Rossi, and S. De Silvestri. Chem. Phys. Lett., 345(12):33, 2001

${ }^{16}$ E. Allard, J. Cousseau, J. Orduna, J. Garin, H. X. Luo, Y. Araki, and O. Ito. Phys. Chem. Chem. Phys., 4(24):5944-5951, 2002.

${ }^{17}$ D.L. Coffen, J.Q. Chambers, D.R. Williams, P.E. Garrett, and N.D. Canfield. J. Amer. Chem. Soc., 93:2258-2268, 1971.

${ }^{18}$ F. Wudl, A. A. Kruger, M. L. Kaplan, and R. S. Hutton. J. Org. Chem., 42:768, 1977.

${ }^{19}$ E. M. Engler, B. A. Scott, S. Etemad, T. Penney, and V. V. Patel. J. Am. Chem. Soc., 99(18):5909, 1977.

${ }^{20}$ Daniel J. Sandman, Timothy J. Holmes, and Diane E. Warner. J. Org. Chem., 44(5):880-2, 1979.

${ }^{21}$ R. Pou-Amerigo, E. Orti, M. Merchan, M. Rubio, and P. M. Viruela. J. Phys. Chem. A, 106(4):631-640, 2002.

${ }^{22}$ R. Pou-Amerigo, P.M. Viruela, R. Viruela, M. Rubio, and E. Orti. Chem. Phys. Lett., 352(5-6):491-498, 2002. TY - JOUR.

${ }^{23}$ R. Andreu, J. Garin, and J. Orduna. Tetrahedron, 57(37):78837892, 2001.

${ }^{24}$ J. Fabian, L. A. Diaz, G. Seifert, and T. Niehaus. J. Molec. Struct.-Theochem, 594:41-53, 2002.

${ }^{25}$ R. Gleiter, E. Schmidt, D. O. Cowan, and J. P. Ferraris. J. of Elect. Spec. and Rel. Phen., 2(2):207, 1973.

${ }^{26}$ A.J. Berlinsky, J.F. Carolan, and L. Weiler. Can. J. of Chem., 52:3373-3377, 1974.

${ }^{27}$ N. Sato, G. Saito, and H. Inokuchi. Chem. Phys., 76(1):79, 1983.

${ }^{28}$ D. L. Lichtenberger, R. L. Johnston, K. Hinkelmann, T. Suzuki, and F. Wudl. J. Am. Chem. Soc., 112(9):3302, 1990.

${ }^{29}$ V. Blanchet P. Hemberger A. Bodi P.M. Mayer, D. Staedter. J. Phys. Chem. A, 117:2753, 2013.

${ }^{30}$ J Fabian. Theor. Chem. Accounts, 106(3):199-217, 2001.

${ }^{31}$ G. Theodorakopoulos W. Kloppe I.S.K. Kerkines, I.D. Petsalakis. J. Chem. Phys., 131, 2009.

${ }^{32}$ G. R. Burns, F. Favier, D. J. Jones, J. Roziere, and G. J. Kearley. J. Chem. Phys., 119(9):4929-33, 2003.

${ }^{33}$ V. Blanchet S.T. Pratt N. Thire, R. Cireasa. Phys. Chem. Chem. Phys., 12(48):15644 - 15652, 2010.

${ }^{34}$ H. Tsubomura and T. Sakata. Chem. Phys. Lett., 21(3):511, 1973.

${ }^{35}$ K. Fuke and S. Nagakura. J. of Mol. Spect., 64(1):139, 1977.

${ }^{36}$ G. Turri B. Chatel G. Girard I.A. Garcia I. Wilkinson B.J. Whitaker V. Blanchet, K. Raffael. The J.Chem. Phys., 128(16):164318, 2008.

${ }^{37}$ P. Piecuch, J.A. Hansen, D. Staedter, S. Faure, and V. Blanchet. J. of Chem. Phys., 138(20):201102-4, 2013.

${ }^{38}$ S. B. Nielsen, M. B. Nielsen, and H. J. A. Jensen. Phys. Chem. Chem. Phys., 5(7):1376-80, 2003.

${ }^{39}$ N. Thire, D. Staedter, R. Cireasa, V. Blanchet, and S. T. Pratt. Phys. Chem. Chem. Phys., 13(41):18485 - 18496, 2011.

${ }^{40} \mathrm{~S}$. A. Trushin, W. Fuss, W. E. Schmid, and K. L. Kompa. J. Phys. Chem. A, 102(23):4129-4137, 1998.

${ }^{41}$ W. Fuss, K. K. Pushpa, W. E. Schmid, and S. A. Trushin. J. Phys. Chem. A, 105(47):10640-10645, 2001.

${ }^{42}$ N. Thire, R. Cireasa, V. Blanchet, and S.T. Pratt. Phys. Chem. Chem. Phys., 12(48):15644-15652, 2010. 